\title{
Significados plurales del dinero estatal Una aproximación etnográfica a los programas sociales de transferencias monetarias.
}

\section{Martín Hornes ${ }^{1}$}

\author{
1Centro de Estudios Sociales de la Economía (CESE) - Instituto de Altos Estudios Sociales (IDAES) de la \\ Universidad Nacional de San Martín (UNSAM). CABA, Argentina. \\ Correo electrónico: m_hornes@hotmail.com
}

Recibido:

diciembre de 2019

Aceptado:

julio de 2020

doi: 10.34096/runa.v41i2.7964

\section{Resumen}

Este artículo se propone abordar los múltiples significados que posee el dinero entregado a partir de los programas sociales de transferencias monetarias. Para tal fin, abordaremos a los diferentes actores sociales involucrados en los circuitos de las políticas de entrega de dinero: a) analizaremos los conocimientos que movilizan los saberes expertos en el diseño de las políticas públicas para otorgar cierto significado al dinero transferido; y b) nos aproximaremos a una experiencia de abordaje etnográfico en torno a la aplicación de un programa social en la escala local, para analizar las contiendas de significados existentes entre los actores locales estatales involucrados en su implementación y los hogares titulares de derechos. Dicho recorrido nos permitirá observar aquello que hemos denominado como la producción social del dinero de las transferencias monetarias: significados del dinero producidos a partir de la relación entre los múltiples actores involucrados, sus saberes y prácticas diversas.

\section{Plural meanings of state money: an ethnographic approach to social cash transfer programs}

\begin{abstract}
This article intends to address the multiple meanings that have the money transferred from the social cash transfer programs. For this purpose, will address the different social actors involved in the circuits of delivery of money policies: to) analyze the knowledge that mobilize the knowledge of experts in the design of public policies to grant certain meaning money transferred, and, b) us approximations to an experience of ethnographic approach around the implementation of a social program at the local level, to analyse the contentions
\end{abstract}

\section{Palabras clave}

Transferencias monetarias: Dinero; Significados sociales; Saberes; Producción social

\section{Key words}

Cash transfers; Money; Social meanings; Knowledge; Social production 
of existing meanings between State local stakeholders in their implementation and rights holders households.

This journey will allow us to observe what we have called as the social production of money from money transfers: meanings of money produced from the relationship between the multiple actors involved, their knowledge and practices different.

\section{Significados plurais de dinheiro do estado: uma abordagem etnográfica para programas sociais de transferência de renda}

\section{Resumo}

Palavras-chave

Transferências de dinheiro; Dinheiro; Significados ociais; conhecimentos; Produção 1. Reemplazaré dimensiones
institucionales, identidades personales y las referencias sobre el territorio donde se desarrolló el trabajo etnográfico por nombres ficticios, en vistas a conservar la identidad de los informantes.

2. El municipio de Avellaneda es la primera localidad lindante con la Ciudad Autónoma de Buenos
Este artigo tem como objetivo abordar os múltiplos significados do dinheiro transferido dos programas de transferência de dinheiro social. Para isso, abordaremos os diferentes atores sociais envolvidos nos circuitos da política de entrega de dinheiro: a) analisaremos o conhecimento mobilizado por especialistas em conhecimento na concepção de políticas públicas para dar algum significado ao dinheiro transferido e, b) abordaremos uma experiência de abordagem etnográfica em torno da implementação de um programa social em nível local, analisar os conflitos de significados entre os atores estatais locais envolvidos em sua implementação e as famílias que defendem a lei.

Essa rota nos permitirá observar o que chamamos de produção social de dinheiro transfere dinheiro: significados de dinheiro produzido a partir da relação entre os múltiplos atores envolvidos, seus conhecimentos e diversas práticas.

\section{Introducción}

En agosto del año 2008, exactamente un año antes de obtener el título de licenciado en Trabajo Social logré insertarme laboralmente en un programa de transferencia monetaria municipal denominado Jóvenes, ${ }^{1}$ perteneciente al Municipio de Avellaneda. ${ }^{2}$ Este estaba dirigido a adolescentes de entre 12 y 18 años de edad que se encontraran en situación de vulnerabilidad social, con el objetivo de que finalizaran sus estudios secundarios y participaran -a contraturno de la escuela- en talleres de apoyo escolar, capacitación y oficios. A condición de cumplir con estos requisitos previamente especificados, los adolescentes recibirían un estipendio mensual en forma de beca que ascendía a la suma de $\$ 150$ (para la época, un valor aproximado a los 49 dólares estadounidenses).

$\mathrm{Al}$ insertarme laboralmente en el Jóvenes descubrí que la emergencia del dinero sorprendía mis esquemas aprehendidos en el campo del trabajo social: las preocupaciones profesionales estaban centradas en las políticas sociales, pero el dinero no era un objeto presente en las reflexiones. Durante mis primeros días de trabajo experimenté cierto extrañamiento y una incomodidad particular a la hora de referirme al dinero en los espacios de intervención social. 
Al cabo de un tiempo observé que los directivos de la Subsecretaría de Inclusión Social de la cual dependía el programa Jóvenes también se mostraban muy preocupados por ocultar la presencia del dinero. Constantemente insistían sobre la utilización de la palabra "plan": "no es un plan, es un programa", para alejarse así de ciertas representaciones negativas de los directivos sobre los planes sociales que transferían dinero. Además, señalaban que este debía ser considerado como "un medio para, y no un fin en sí mismo"; apelaban a esa definición del dinero por considerarla como "la mejor estrategia para que los adolescentes participen del programa".

Paradójicamente, para todo un universo de funcionarios y agentes locales estatales (trabajadores sociales y planificadores de política pública), mientras más se usaba el dinero en las políticas sociales, más se intentaba silenciarlo y ocultarlo.

Tales estrategias de ocultamiento se contrastaban con un rumor permanente en los barrios populares de Avellaneda: todos hablaban del dinero del programa. Los adolescentes se referían a él como "una bocha de guita", y así consideraban no solo su cantidad sino su cualidad: el acceso a prácticas de consumo significativas para ellos (ropa, zapatillas, celulares, salidas nocturnas, etcétera). En los presupuestos de los hogares, el dinero del Jóvenes ocupaba un lugar significativo: su presencia marcaba distinciones frente a otros ingresos -"es como un sueldito", señalaban algunos padres-y generaba controversias $\mathrm{y}$ tensiones entre padres e hijos, esposas y maridos. Incluso, la presencia del dinero estatal movilizaba juicios y evaluaciones en el ámbito comunitario: "les pagan para que se droguen y salgan a robar", mencionaban ciertos vecinos.

Siete años más tarde me encontraba en una de las oficinas de la sede del Banco Mundial en la República Argentina. Tenía la posibilidad de entrevistar a un destacado experto en políticas sociales de transferencia de dinero de Latinoamérica y, quizá, uno de los más relevantes en el surgimiento de dichas políticas en Argentina. "Es algo simple", señalaba nuestro entrevistado: "no hay una reflexión sistemática sobre el dinero": "El dinero es un medio de cambio para adquirir bienes y servicios".

Desde aquel momento pude comenzar a comprender que muchas de las dificultades para interpretar lo que sucedía en aquellos espacios profesionales vinculados al trabajo social guardaban relación con una trama mucho más amplia de actores sociales, saberes y significados acerca del dinero transferido a partir de los programas sociales de transferencia monetaria (TM).

Las viñetas etnográficas que acabamos de recorrer nos aproximan al objetivo central del artículo: reconstruir la producción social de los significados del dinero proveniente de los programas sociales de TM en la Argentina reciente (2008-2015). Se trata de indagar sobre la multiplicidad de tramas y sentidos asociados al dinero estatal analizando sus significados sociales y morales en los circuitos de las políticas sociales de TM. ${ }^{4}$

Durante los últimos veinte años, los programas sociales de TM se consolidaron como las principales formas de combate a la pobreza en la región de América Latina y El Caribe. En la actualidad, estas intervenciones se encuentran presentes a escala mundial y guardan similitudes: a) suplantan la tradicional provisión de bienes y servicios por la entrega de dinero en efectivo a los sectores más vulnerables; $\mathrm{y} b$ ) establecen condicionalidades en materia de salud
3. A lo largo del texto usaré comillas para identificar las palabras de los entrevistados y las categorías nativas que son objeto de reflexión etnográfica.
4. La bibliografía sobre la temática se refiere a las políticas de entrega de dinero bajo distintas acepciones. Utilizaremos la denominación genérica de transferencias monetarias para apartarnos de las distinciones programáticas y denominaciones específicas, dado que nuestro interés reviste en los significados sociales que adquiere el dinero. 
y educación sobre los menores pertenecientes al hogar (controles sanitarios y asistencia escolar) (Hornes, 2020).

En diferentes trabajos hemos explorado las características que han asumido las TM en los países latinoamericanos, para destacar las particularidades del caso argentino, y señalar debates expertos y condiciones programáticas generales (Hornes y Maglioni, 2020). En este trabajo, nos interesa aproximarnos a una perspectiva poco explorada que indague sobre la trama de significados sociales que adquiere el dinero entre los distintos actores involucrados en las políticas de TM. Aquello que hemos denominado como la producción social de las TM: prestando atención a los saberes expertos en políticas sociales, a los actores locales estales involucrados en la implementación de las políticas y a las prácticas monetarias de los hogares titulares de derechos, analizaremos los significados sociales y morales que adquiere el dinero distribuido a partir de las TM estatal (Hornes, 2020).

Hablar de significados sociales del dinero implica desasirse de aquellas nociones "apriorísticas" que piensan al dinero como un objeto propio de relaciones mercantiles, impersonales y afectivamente neutras. A partir de mediados del siglo XX, desde el campo antropológico y sociológico se construyeron distintas narrativas que reflexionaron en torno a la presencia del dinero antes y más allá de los mercados y las relaciones mercantiles, y sobre la existencia de monedas múltiples (Bloch, 1989; Bloch y Parry, 1989; Dodd, 2014; Weber y Dufy, 2009). Desde mediados de la década del ochenta asistimos a una renovada agenda de investigación sobre los estudios sociales del dinero, que proviene de disciplinas tan diversas como la economía e historia económica (Théret, 2007; Fontaine, 2008; Roig, 2016), la filosofía (Cuillerai, 2004), la antropología (Guyer, 1994, 2004) y la sociología (Zelizer, 2009 y 2011).

La aparición de la obra de Viviana Zelizer titulada Los significados sociales del dinero (2011) representó un nuevo punto de ruptura sobre tales interpretaciones. Zelizer (2009 y 2011) demostró los múltiples significados personales, sociales y morales que pueden acompañar las transferencias de dinero. Sus hallazgos muestran cómo el dinero puede introducir distinciones, disputas, negociaciones y evaluaciones incluso en las relaciones de mayor confianza e intimidad y, en determinados casos, empuja a sus participantes a establecer límites específicos para garantizar diferentes transferencias. Otros trabajos resaltaron también la dimensión más experiencial o sensible del dinero al destacar que la pluralidad de significados explorada por Viviana Zelizer resulta un sustrato esencial para comprender la heterogeneidad de representaciones que acompañan al dinero, y la multiplicidad de significaciones y usos sociales que adquiere este en la vida social (Guyer, 2004; Weber y Dufy, 2009).

Dialogaremos con los trabajos de Ariel Wilkis (2013), dado que ha explorado con detenimiento una multiplicidad de prácticas económicas y significados del dinero en los sectores populares, y demostró las múltiples conexiones y significados que el dinero puede alcanzar entre actores supuestamente disímiles. Una nueva infraestructura monetaria (Wilkis, 2014) que atraviesa al mundo popular devela, no solo la falsa representación de que los pobres se encuentran desmonetizados, sino también que los múltiples significados sociales y morales que transporta el dinero entre y hacia los sectores populares aportan elementos clave para explorar e interpretar concepciones sobre el orden social en términos de estatus, jerarquías y relaciones de poder (Wilkis, 2017). 
Nuestro trabajo se suma a tales interpretaciones e incorpora un punto de vista que nos permite dar cuenta de la producción social de los significados del dinero de las TM estatales: demostraremos que se trata de un dinero que se compone a partir de la intervención y el entramado de múltiples saberes y significados. En ese sentido, exploraremos los circuitos de las políticas sociales prestando atención a las definiciones sobre el dinero que difunden los saberes expertos en TM pero deteniéndonos, a su vez, en las reinterpretaciones que se suceden en instancias de gestión cotidiana de los programas sociales estatales para analizar los significados sociales y morales movilizados por los titulares y hogares receptores de TM. ${ }^{5}$

Este artículo expone algunos de los hallazgos de mi tesis doctoral (Hornes, 2018). Parte del material que sustentará nuestro análisis proviene de entrevistas en profundidad realizadas a expertos en TM (funcionarios de organismos internacionales, regionales y estatales, especialistas en políticas sociales, expertos del tercer sector -ONG/ fundaciones- etc.). ${ }^{6}$ Además, nuestro trabajo se nutre del abordaje etnográfico centrado en los procesos de implementación del programa Jóvenes, el cual nos permite profundizar en las interpretaciones de los actores locales estatales y de los titulares de derechos acerca de los significados del dinero estatal. ${ }^{7}$

El artículo se organizará de la siguiente manera. En el primer apartado, exploraremos las concepciones sobre el dinero entre los saberes expertos en programas de TM para visibilizar conocimientos que dichos actores ensamblan para diseñar el dinero de las políticas sociales. En el segundo apartado, describiremos y analizaremos eventos significativos en la gestión cotidiana del Jóvenes, para explorar controversias entre los actores locales estatales involucrados en la implementación de la TM municipal y los hogares titulares acerca de los significados sociales que adquiere el dinero. En un tercer y último apartado, reflexionaremos en torno a la producción social de las TM y su contribución al campo de las políticas sociales.

\section{Significados expertos sobre el dinero de las TM}

Los economistas lo vemos desde un punto de vista más simple. Hace un tiempo estaba por dar una charla en una conferencia, me estaba poniendo un traje, y mi hija de tres años me pregunta; ¿De qué vas a hablar papi? Le respondo: viste que a veces vemos gente en la calle, que no tiene plata, voy a hablar sobre la gente y qué se puede hacer. Ella dice: ah, ya sé qué se puede hacer, darles plata. La primera aproximación de los economistas es un poco la de mi hija: darles plata. Ahí te diría que ni siquiera hay una reflexión sobre el tema, sino que es más bien ir y darles plata, con base en los trabajos e investigaciones de otra gente que dicen que es mejor dársela a las mujeres. (Entrevista a experto local, 15 de abril de 2015)

Estas palabras conforman un fragmento de entrevista realizada a uno de los expertos más destacados en políticas sociales de TM de la República Argentina. Se trata no solo de uno de los referentes más importantes de la temática, sino también de uno de los centros de investigación de mayor influencia en Argentina en el campo actual de las políticas de entrega de dinero. Consultor de organismos internacionales, regionales, entes estatales, y demás atributos expertos, sus palabras generaban para mí una gran expectativa.

Pasados unos meses de trabajo de campo entre expertos en TM de nuestro país, estas referencias sobre el dinero seguían sucediendo. Los expertos
5. Utilizaremos la definición de saberes expertos elaborada por Morresi y Vommaro (2011). Para el caso de la denominación de actores locales estatales, seguimos la definición de los estudios antropológicos sobre Stategraphy (Thelen, T., Vetters, L. y Von BendaBeckmann, K. (2014).

6. La extensión de este artículo no nos permite presentar a los actores expertos y sus credenciales de expertise. Sin embargo, la selección de testimonios que utilizaremos reúne a los expertos más destacados en el campo de instituciones de expertise en TM argentinas y a los actores más relevantes pertenecientes a los organismos internacionales.

7. El trabajo de campo etnográfico que da lugar a dicha sección tuvo lugar entre los años 2008 y 2011. 
entrevistados referían al dinero como algo "simple", "para intercambiar en el mercado" o "un elemento para que las familias tomen las mejores elecciones sobre sus formas de desarrollo". Observaba estas definiciones con gran preocupación, porque en ninguna de ellas existía una reflexión acerca de la naturaleza del dinero. Luego de un tiempo y recurriendo a los aportes de la sociología económica (Orleán, 2007; Théret, 2007), comprendí que tales interpretaciones formaban parte de las concepciones de la economía moderna y la teoría económica clásica y neoclásica, de acuerdo con las cuales el dinero se ha presentado desde una visión instrumental y estandarizada, como un elemento homogéneo y cualitativamente neutro, ligado a sus funciones unidad de cuenta, medio de cambio y reserva de valor (Blanc, 2009).

Considerando lo anterior, nuestra hipótesis de trabajo será la siguiente: las concepciones de los expertos en TM arrastran y llevan consigo las premisas que porta el dinero en el campo económico, y utilizan tales cualidades generales del dinero para organizar el diseño de una moneda específica de las políticas sociales. Observaremos cómo los saberes expertos ensamblan una serie de conocimientos en torno a las TM: un universo de actores que reproducen significados económicos sobre el dinero y, de forma simultánea, actúan diseñando y programando una moneda específica a ser transferida a partir de las políticas sociales. A continuación, nos detendremos en algunas de las premisas centrales que estructuran los programas de TM.

\section{Capitales e inversiones}

Los expertos proponen pensar las TM como condición de que los hogares realicen inversiones en el capital humano de los menores pertenecientes al hogar en aspectos educativos y de salud, con el objetivo de quebrar la pobreza que se transfiere de una generación a la siguiente. Así los sintetizaba uno de nuestros entrevistados:

Algunos programas alcanzan a generar dos años más de escolaridad, que no es poco, pero si estás hablando como la promesa que tuvieron muchos de estos programas de ser una herramienta de reducción de la pobreza intergeneracional, no es mucho. Solo promueven la educación y la salud: no hay nada que hayamos podido confirmar en el largo plazo. (Entrevista a experto del Banco Interamericano de Desarrollo - Área de Protección Social, 2 de julio de 2015)

La cita de entrevista precedente nos aproxima a aquello que Barba Solano (2011) ha denominado como los "falsos espejismos" que se han construido en torno a las TM. En base a un análisis exhaustivo de evaluaciones realizadas sobre diferentes programas de TM de la región de América Latina, el autor ha señalado que las transferencias han tenido un efecto notable en el descenso de la intensidad de la pobreza. Las mismas y constantes fuentes que han contribuido a legitimar a las TM y a convertirlas en un paradigma a seguir han demostrado el desmoronamiento de las premisas centrales que sostienen a la noción de capital humano: a) las evaluaciones e investigaciones recientes muestran débil incidencia en la pobreza en corto plazo; b) las mejoras en el salud y en la alimentación no demuestran una contrapartida directa en la educación; y c) las mejoras en los niveles educativos tampoco garantizaron una acumulación sustancial de capital para el desempeño en el mercado de trabajo (Barba Solano, 2011).

La teoría del capital humano se origina dentro del campo de la teoría económica neoclásica, y de la mano de referentes como Gary Becker, representante del 
monetarismo y de las teorías de las expectativas racionales del Departamento de Economía de la Universidad de Chicago, Estados Unidos (Lavinas, 2017). La premisa básica del capital humano -aporte que le valió el Premio Nobel de Economía en el año 1992 a Becker- sostiene que la inversión directa que los individuos realicen en ciertos aspectos -como, por ejemplo, educación, entrenamiento laboral, salud y nutrición-incidirán de forma directamente proporcional sobre sus competencias y su capital económico futuro (Becker, 2008).

Categorías como el capital humano resultan un elemento teórico que modela al dinero de las políticas sociales. Más allá de la falta de un sustento empírico para indicar sus resultados, o de la imposibilidad de demostrar que esa categoría económica cabe en la realidad social, dentro de los saberes expertos el capital humano representa un conjunto de discursos lógicos basados en una serie de hipótesis que se presentan como irrefutables, independientemente de que eventualmente sucedan o no. En este sentido, la noción de inversión en el capital humano prescribe al dinero en un doble movimiento: por un lado, lo define como pequeños montos que viabilizan mejoras en el consumo y el bienestar de los pobres; mientras que, por otro lado, diseña una moneda que proyecta la realización de las promesas de desarrollo que contienen las políticas dentro de un futuro distante (Dapuez, 2013).

\section{Condiciones sobre el dinero}

Pese a que la noción de capital humano se ha transformado en una "promesa de reducción de la pobreza" con pocos resultados comprobables o con "resultados finales" que han tenido "efectos modestos" (Banco Mundial, 2009, p. 22), las condicionalidades juegan un rol fundamental en la construcción de discursos de legitimidad política y social de las TM, y los expertos las difunden ampliamente en el repertorio de aspectos programáticos.

Dentro de la literatura, los expertos han caracterizado TM que contienen una "condicionalidad fuerte", y otra serie de iniciativas que refieren a "condicionalidades blandas" (Cecchini y Martinez, 2013). En el universo de las "condicionalidades fuertes" se ubican los programas PROSPERA (México) y Familias en Acción (Colombia), los cuales establecen una relación directa entre la noción de TM y condición, con lo que ejercen un control sistemático y punitivo sobre su cumplimiento. Argentina forma parte, junto con el programa Bolsa Familía de Brasil, de los países que ejercen "condicionalidades blandas" sobre las TM. Considerando las acepciones expertas, el incumplimiento de dichas condicionalidades no suele ser considerado como sancionatorio o, en algunos casos, la verificación de ellas tiende a ser débil o inexistente. De la siguiente manera daba cuenta de esta concepción uno de los expertos con mayor trayectoria regional en la República Argentina:

Tenés el modelo mexicano y el modelo brasileño. El mexicano es un tema muy de rational choice (elección racional): si vos no cumplís, como esto tiene que generar un cambio de comportamiento, yo te lo saco y te demuestro que tengo un poder punitivo. En Brasil, que tiene un enfoque más del derecho, el no cumplimiento de la condicionalidad activa al Estado nacional y al municipio para entender por qué no estás cumpliendo. Básicamente, Argentina eligió el modelo de Brasil acerca de transferir ingresos, pero nunca se implementó ningún tipo de control siquiera para el seguimiento de las familias. (Entrevista a experto local, 15 de julio de 2015)

Los programas de TM fueron diseñados en torno a los principios que marcaron la agenda de los organismos internacionales durante la década de los noventa: 
las nociones de participación, empoderamiento y corresponsabilidad. Las "condicionalidades" reúnen o sintetizan tales aspectos, y una serie de trabajos han resaltado los efectos de estas políticas en tanto medidas que empujan a los sujetos a participar activamente en su desarrollo y ser proveedores de su propio bienestar (Molyneux, 2009); como políticas que inducen o moldean los comportamientos de los pobres (Cotta y Paiva, 2010) o transforman cuestiones de derechos en medidas punitivas (Lo Vuolo, 2013).

Las perspectivas anteriores resultan relevantes porque se constituyen sobre la base de trabajos de investigación de otras experiencias de TM de la región. Sin embargo, para la situación de la República Argentina en particular, señalan una paradoja: Argentina se ubica entre las propuestas de TM con "condicionalidades blandas" y, además, muchos de nuestros entrevistados señalaron la inexistencia de medios de control sobre las condicionalidades. Entonces, podríamos inferir que parte del establecimiento de las condicionalidades remite en la operación de trasladar algunas de las propiedades que los expertos le atribuyen de "forma natural" al dinero:

Si yo te doy plata, vos cambiás tu comportamiento. Si te doy mucha plata vas a cambiar mucho tu comportamiento. Si te doy poquita, vas a cambiarlo poquito. Eso no vale la pena discutirlo. Va a pasar. Si yo le doy a cada señora diez mil dólares por cada niño todos los meses, va a tener un cambio de comportamiento muy importante. Y si le doy un peso, no se va a dar por enterada. Si le doy $400,600,800$, va a cambiar algunas cosas. (Entrevista a experto del Banco Mundial - Área de Protección Social, 22 de junio de 2015)

Las palabras de nuestro entrevistado ratifican algunas de las apreciaciones que los expertos sostienen: el dinero como un elemento homogéneo que contiene significados unívocos. Desde los saberes expertos y las prácticas de expertise que los economistas movilizan por medio de las entregas de dinero, las condicionalidades solo pueden tener un sentido uniforme, en tanto interpretan que los agentes van a realizar cambios en sus comportamientos acordes con la supuesta naturaleza de la moneda. La introducción del dinero supone para los expertos que los hogares pobres van a realizar conductas y prácticas racionales, incluso considerando los montos transferidos como un elemento especifico de cálculo entre costos y beneficios.

La extensión de este trabajo no nos permite explorar la totalidad de operaciones materiales y no materiales que realizan los expertos sobre el dinero de las TM, entre las que podríamos desarrollar la titularidad femenina, el uso de tecnologías diversas, monitoreos y evaluaciones e, incluso, procesos de experimentación en la escala real (Hornes, 2019). Sin embargo, nos permite plantear una aproximación: las premisas que enunciamos en estas páginas y los conocimientos que los saberes expertos movilizan no hacen más que trasladar nociones propias del campo económico sobre las políticas sociales, y así colaboran en el diseño de una moneda específica a ser distribuida por las nuevas tecnologías monetarias de pago a la asistencia que representan las TM.

Como postula nuestra perspectiva, el dinero es un producto social maleable (Zelizer, 2017). A partir de las páginas siguientes, exploraremos eventos significativos en la implementación del programa Jóvenes para contemplar las dos caras de una misma moneda: el dinero de las TM contiene una matriz diseñada por los saberes expertos pero, a su vez, posee una vida social que desborda tal diseño. 


\section{Eventos de la gestión cotidiana}

\section{Producción de condiciones en Villa Asunción}

"Ahí va el chico del plan de los 150 (pesos)", eran palabras que solía escuchar en boca de los vecinos de Villa Asunción. Así se referían a mi rol como trabajador social y específicamente a mi persona como principal responsable de la instancia de admisión al Jóvenes. A modo de respuesta, y recurriendo a algunas de las fundamentaciones establecidas por el programa, enfatizaba la noción de "beca" para referirme al conjunto de condiciones que debían cumplir los adolescentes para percibir el dinero.

Palabras y escenas como las anteriores se sucedían cotidianamente. Infinidad de veces era interceptado en el barrio e indagado respecto de las posibilidades de ingreso al Jóvenes. Ante la constante demanda, yo solía repetir que debían cumplir con una serie de pasos sucesivos: la inscripción en la lista de espera, la realización de una entrevista familiar en el hogar y -ante la eventual existencia de becas- la evaluación del equipo técnico perteneciente al programa. Mediante la puesta en movimiento de estos encuentros se iban conformando las condicionalidades referidas al programa.

Aquellas instancias que contribuían a la construcción y definición de las condicionalidades que debía establecer el Jóvenes no resultaban casuales, sino que estaban completamente incorporadas a los repertorios de intervención de los agentes locales estatales. Formaba parte del denominado proceso de inserción que los trabajadores sociales experimentábamos al momento de sumarnos a los equipos técnicos: una capacitación que preveía el desarrollo de cierta expertise en los "Criterios de admisión y procedimiento del programa Jóvenes" (Hornes, 2017). A continuación, resumiremos parte de dichas instancias y sus cualidades particulares para producir determinadas condiciones sobre el dinero transferido.

Durante la instancia de inscripción a la lista de espera, solían reforzarse algunos de los aspectos referidos a las condicionalidades del programa. En dicha etapa, se recordaban las condiciones de escolaridad y asistencia al Jóvenes, a la vez que se registraban los datos generales de los adolescentes (nombre y apellido, dirección, escuela a la que asistían, conformación familiar, etc.). También solía indagarse a los adolescentes respecto de los motivos por los cuales estaban interesados en participar del programa.

La visita domiciliara por parte del trabajador social implicaba un eslabón central de la cadena, quizás el más anhelado por los adolescentes y sus grupos familiares. Estos encuentros tenían una duración aproximada de 45 minutos, y en ellos se priorizaba la presencia conjunta del adolescente y su madre o padre. Normalmente, se iniciaban con una precisa definición de la pertenencia institucional del programa y sus principales líneas de acción. Se enfatizaba que el programa era una iniciativa de la Subsecretaría de Inclusión Social del municipio y que estaba dirigido a adolescentes de entre 12 y 18 años que atravesaban problemas en su tránsito por el sistema educativo formal, los cuales recibirían una beca de $\$ 150$ por su participación.

El encuentro proseguía con una enumeración detallada de las condicionalidades del Jóvenes. En primer lugar, se introducía la condición de la escolarización de los adolescentes como uno de los requisitos fundamentales, ya fuera para garantizar la continuidad o la reinserción al sistema educativo formal. En segundo lugar, se mencionaba la asistencia a talleres educativos y recreativos 
brindados en las instalaciones del programa, a los cuales los adolescentes debían asistir a contraturno de la escuela. Ambas condiciones eran fundamentales y complementarias, e incluso existían sanciones económicas ante el eventual incumplimiento de algunas de ellas. Estas se traducían en descuentos sobre el monto de la beca. En los casos en que el incumplimiento persistiera, se produciría la baja del programa y el retiro de la beca.

Una vez finalizada la etapa ritual de presentación del programa y la enumeración de las condiciones, la visita continuaba con la indagación de aspectos referidos al denominado "informe social". Mediante la aplicación de una entrevista semiestructurada, se abordaban aspectos referidos a la composición del grupo familiar, la situación económica, habitacional, educativa y de salud del hogar. Estos aspectos eran considerados como fundamentales, ya que el esquema de aplicación del programa contaba con un "índice de vulnerabilidad" que preestablecía tres niveles que definían el menor o mayor grado de criticidad del hogar (Hornes, 2017). Además, poseía un apartado final en el cual cada trabajador social podía volcar sus apreciaciones personales, y especificar el clima/ contexto de entrevista, verbalizaciones significativas de los entrevistados, relevancia de alguna problemática detectada, etcétera.

Una vez abordados todos los ítems que establecía el informe social, el encuentro finalizaba con una aclaración particular. Dado que en algunas ocasiones la lista de espera de inscripción al programa alcanzaba a más de 80 adolescentes, se reiteraba que el objetivo principal del programa era incorporar a aquellos jóvenes que se encontraban en situación de vulnerabilidad social, y destinar las becas disponibles a quienes se encontraran en mayores niveles de criticidad dentro de aquel grupo.

La serie encadenada de instancias que aquí presentemos resulta ilustrativa, no solo por ser eventos que tenían un rol preponderante en la producción y reproducción de la política pública a nivel barrial sino que, además, permiten demostrar el incesante empeño que los agentes locales estatales realizábamos por encarnar los significados del dinero diseñado por los saberes expertos en TM. Cada uno de esos eventos significativos junto a los candidatos se convertían en instancias rituales por medio de las cuales los agentes locales estatales encarnábamos el dinero producido por los expertos en los laboratorios virtuales de diseño de las TM: reforzábamos aspectos constitutivos de la noción de acumulación del capital humano esgrimiendo las condicionalidades programáticas.

Como veremos a continuación, pese a los esfuerzos que realizábamos los actores locales estatales, la vida social del dinero desbordaba cualquier tipo de intento de uniformidad experta: los titulares y hogares receptores cuestionaban las condiciones de las transferencias y negociaban los significados del dinero recurriendo a saberes prácticos e ideas ordinarias ancladas en sus contextos particulares y sus necesidades sociales específicas.

\section{Controversias sobre los significados del dinero.}

“¿Todo esto por 150 pesos?", repetía "Lolo" incesantemente para quejarse de las cosas que tenía que hacer en los talleres en los que participaba a lo largo de la tarde. Con 17 años, "Lolo" cargaba con una serie de experiencias delictivas y uso frecuente de drogas. 
Luciano, más conocido como "Lolo", vivía con su mamá, Carmen -de unos 40 años- y su hermano Nahuen, de 12 años. Con ellos convivía Julián, el novio de Carmen, y el hijo de ambos: Máximo, un bebé de apenas 8 meses. Nahuen y Luciano eran hijos del primer matrimonio de Carmen.

A inicios del año 2009, y ante la necesidad de escolarizar a un grupo de adolescentes dentro de un esquema de educación formal, "Lolo" se incorporó en el marco de acciones y en las instalaciones del programa a un ciclo de formación denominado Centro de Escolarización Secundaria para Adultos y Jóvenes (CESAJ). Dependiente del Ministerio de Educación de la Provincia de Buenos Aires, se trataba de una modalidad educativa especial para trabajar con grupos sociales vulnerables.

Durante el ciclo lectivo del año 2009 la participación de "Lolo" en el espacio educativo presentó interrupciones. Con frecuencia solía faltar a clase sin justificación; en ocasiones lo hacía para realizar algún trabajo informal, o tenía algún tipo de impedimento familiar para asistir (por ejemplo: tareas de cuidado u organización doméstica). Sin embargo, durante el mes de octubre "Lolo" se ausentó tres días seguidos y circulaba un rumor preocupante: "comentan que está en cama por un balazo en la pierna".

Inmediatamente, el coordinador del programa me encomendó la visita a la casa de la familia para averiguar de qué se trataba. Luego de varios intentos frustrados, recibí una llamada telefónica de su mamá a mi celular personal. "No sé qué hacer", fueron sus primeras palabras, y agregó: "tengo a Lolo en la casa de mi mamá con una bala en la pierna".

En pocos minutos logré llegar a la casa de Irma, la abuela de Luciano. Me acompañó hasta la habitación donde él estaba. Apenas entré, "Lolo" se reincorporó sobre la cama, me miró a los ojos y me dijo: "Me lastimé con una trucheta, ${ }^{8}$ Tincho. Ayer fui a robar a unos chinos sobre (la calle) Pergamino cerca del Cementerio"; así se ahorró cualquier tipo de preámbulo innecesario. Mientras me acomodaba en una silla próxima a la cama, agregó: "los pibes con los que fui me dejaron re-tirado. Yo me asusté porque pensé que el chino de la puerta estaba enfierrado [portaba un arma] y tiré. Pero la trucheta reventó mal".

"Lolo" tenía varias lastimaduras provocadas por perdigones en el muslo de la pierna derecha. Carmen le había limpiado la herida varias veces con agua oxigenada, pero resultaba necesario acercarse a algún centro de salud. Toda la situación generaba una serie de inconvenientes. En primer lugar, desde mi función como trabajador social estaba obligado a comunicar la situación a mi superior y a la dirección del programa. En segundo lugar, era probable que la dirección del programa realizara alguna intervención que expusiera legalmente la situación de "Lolo". En tercer lugar, Carmen imaginaba algunos de estos escenarios, y antes de que finalizara nuestro contacto telefónico me sugirió: "por favor, no lo cuentes a nadie hasta que no sepamos que hacer".

Durante el transcurso de esa tarde se sucedieron varios intercambios. "Lolo" y Carmen se mostraban inquebrantables, evitando de cualquier forma el contacto con alguna institución de salud, escenario que dificultaba mi posición y el diálogo con las autoridades del programa. Las autoridades de la Subsecretaría de Inclusión Social insistían sobre el hecho de no querían exponerse a problemas legales por falta de intervención del Jóvenes.
8. "Trucheta" se denomina a un arma de producción casera muy similar a una "Itaca". Posee un caño que se rellena con pólvora y perdigones, y un martillero que facilita la explosión de la carga. 
Pese a que movilizamos distintas estrategias, Carmen y "Lolo" no cambiaban de opinión. Cerca de las 18 horas recibí una llamada telefónica de las autoridades de la Subsecretaría de Inclusión Social: "si la familia no accede, tenemos que desvincularlo a Luciano del programa Jóvenes, porque institucionalmente no podemos evadir esta responsabilidad", señalaron. Al comentarle a Carmen la situación, arrojó: "necesito tiempo para pensarlo, mañana te tengo una respuesta". Decidí otorgarles ese tiempo, lo que me causó problemas con las autoridades del Jóvenes: "era una decisión que no podías tomar sin antes consultar".

A la mañana siguiente, Carmen me recibió con cierta alegría y remarcando que "había encontrado una solución". Mientras nos acomodábamos en la mesa de la cocina, inició su exposición mencionando sus agradecimientos hacia mi persona en particular, para luego desplegar la "solución":

Yo sé que hasta ahora han hecho lo posible para que Luciano no salga perjudicado, pero te voy a decir la verdad... yo no quiero quedarme sin la plata del Jóvenes. Y no es solo la plata, es también la posibilidad de que Nahuen tenga una oportunidad y pueda entrar al programa, no quiero que termine como su hermano.

Carmen expondría "la solución" cómo una estrategia de cambio de titulares en el programa: Nahuen, con sus 12 años ya cumplidos, reemplazaría a Luciano en el Jóvenes. De forma sintética, señalaría a la estrategia como una alternativa mediadora en el conflicto. Mi respuesta empañaría su alegría:

No va a ser posible... nosotros manejamos todo un procedimiento de lo que llamamos 'las altas al programa', es decir, los ingresos de nuevos chicos. Muchos padres piensan estrategias como la que vos proponés, pero hay toda una serie de pasos a seguir. Tenemos una lista de espera con más de 80 chicos del barrio, y cuando surge la posibilidad de incorporar a alguien recurrimos a esa información. Luego hacemos una serie de entrevistas a los adolescentes y visitamos sus hogares para conocer a las familias y sus necesidades, tratando de priorizar que ingresen aquellos que más lo necesitan.

Con dichas palabras sinteticé aquello que, en los esquemas programáticos del Jóvenes, se conocía como los "Criterios de Admisión y procedimiento". Por supuesto que mi "explicación técnica" no solo no coincidía con "la solución" propuesta por Carmen, sino que, además, le resultaba irrisoria: "Perdoname, pero yo no entiendo... Nahuen es un buen alumno en la escuela, tiene la edad para estar en el programa, y vos sabés que nuestra familia también tiene necesidades al igual que otras", arrojó Carmen.

Por varios minutos intenté explicarle los criterios del programa, señalando que se trataba de aspectos considerados por las autoridades del Jóvenes "para otorgar transparencia a los procesos de incorporación y garantizar la igualdad de oportunidades". Carmen se mostraba ahora un poco más enfurecida:

9. Un valor aproximado a los 900 dólares estadounidenses para la época, y que representaba más de veinte veces el valor del dinero transferido en el marco del programa Jóvenes.
Todo muy lindo... pero nuestra realidad es otra. Yo te puedo escuchar hablar toda la mañana sobre cómo ustedes piensan que se deberían hacer las cosas, pero a mí no me resuelve nada. Tengo a Nahuen y cumple con la escuela, mientras que Luciano va al Jóvenes y termina con la pierna llena de perdigones. La plata del Jóvenes no es un dineral pero a nosotros nos sirve. Luciano ya le encontró el gusto a la plata fácil robando... se llevó más de tres lucas (tres mil pesos) de los chinos. ${ }^{9}$ Ya te digo... nuestra realidad es otra. 
Durante el transcurso de ese día y tras la imposibilidad de llegar a un acuerdo, "Lolo" resultaría desafectado del Jóvenes, lo que en la jerga cotidiana los técnicos denominábamos como "una baja". Mi intervención como trabajador social finalizaría con una derivación a un colega vinculado a un programa social de prevención del delito. Carmen sería implacable en nuestra conversación final:

\begin{abstract}
La verdad que... ¿ustedes que es lo que hacen? Cuando tenemos un problema no nos pueden ayudar y la única salida que nos dan es que Lolo no pueda estar más en el programa. Además de que nos perdemos la plata de la beca, tampoco pueden darle la posibilidad a Nahuen. No me queda otra que pensar lo que piensan todos los vecinos del barrio: el Jóvenes termina siendo un depósito de pibes que no estudian y quieren la plata fácil.
\end{abstract}

La escena etnográfica que reconstruimos más arriba, y que tiene a "Lolo" y Carmen como principales protagonistas, resulta significativa porque nos permite introducir la multiplicidad de significados sociales que adquiere el dinero de las TM en los barrios populares. Algunos de los pasajes de la reconstrucción etnográfica nos sirven para ilustrar estos aspectos.

En primer lugar, permite identificar que al interior de los hogares titulares existían significados disímiles sobre el dinero. Para "Lolo", el dinero del Jóvenes significaba un "montón de cosas" que debía hacer en el marco de su participación en el programa, un dinero desjerarquizado frente a los montos que obtenía por la vía delictiva: "se llevó más de tres lucas (tres mil pesos) de los chinos". Para Carmen, la TM no representaba un "dineral" pero "servía": aportaba al presupuesto del hogar y significaba una "oportunidad" para Nahuen.

Desde la mirada de un trabajador social, escenas como las que analizamos muchas veces remitían a problemas sociales y familiares. Movilizando nuestra perspectiva sobre el dinero y las políticas sociales podemos advertir que existen una multiplicidad de tensiones intergeneracionales sobre los significados y usos sociales del dinero transferido: los significados de las TM se dirimen entre madres, padres e hijos, y negocian valores personales, morales y familiares específicos (Wilkis y Hornes, 2017).

En segundo lugar, nos permite constatar cómo los agentes locales estatales estábamos preocupados por transmitir cierto saber experto sobre el dinero y, al mismo tiempo, debíamos lidiar con los cuestionamientos y las negociaciones que los adolescentes y los hogares titulares realizaban sobre los significados de las TM. La "solución" esgrimida por Carmen da cuenta de esta situación, ante la cual los técnicos movilizábamos constantemente categorías que formaban parte de la representación oficial del programa (formas de acceso, requisitos para la inscripción, condicionalidades, etcétera) e implicaban prácticas que reproducían cierto saber experto sobre el dinero (inversiones eficientes en el capital humano).

Siguiendo con lo anterior, y parafraseando a Carmen, podríamos advertir que tales situaciones develaban realidades y soluciones diferentes. Los técnicos parecíamos muy preocupados por marcar el dinero (Zelizer, 2011) de las TM bajo las condiciones de expertise de la "transparencia y la igualdad"; sin embargo, nuestras interpretaciones no cuadraban en la vida cotidiana de las familias. Para Carmen, y contemplando las necesidades económicas y la experiencia de "Lolo", el dinero del Jóvenes significaba no solo un ingreso monetario sino, también, una forma de priorizar el cuidado de la familia y otorgarle una "oportunidad para Nahuen". 
En tercer lugar, las palabras de Carmen reponen también una pregunta sobre la dimensión pública del dinero de las TM (Hornes, 2015b). En nuestra conversación final, y al referirse al Jóvenes como "un depósito de pibes que no estudian y quieren la plata fácil", podemos señalar la presencia de un conjunto de juicios y evaluaciones morales que circulan hacia y entre las clases populares a partir de las TM. Las palabras de Carmen revelan que, más allá de cualquier definición experta o programática preexistente, el dinero de las TM moviliza un capital moral específico (Wilkis, 2017): un conjunto de juicios y evaluaciones morales (valores, virtudes, obligaciones y responsabilidades) que conectan a los diferentes actores y a partir de las cuales se producen jerarquías que permiten considerar o considerarse un titular legítimo de la redistribución monetaria estatal.

\section{Conclusiones}

A lo largo de este artículo, y en el ejercicio de explorar los significados sociales del dinero de los programas de TM, demostramos la capacidad del dinero de actuar como un gran conector social: mirar al dinero nos permitió conectar universos tan distantes como el de los saberes expertos en políticas sociales con las dinámicas monetarias y familiares de los hogares titulares de TM.

Incorporando la mirada de quienes piensan al dinero que forma parte de las intervenciones estatales observamos cómo los saberes expertos aluden constantemente al diseño y a la programación de un dinero específico que lleva inscriptas en su génesis las premisas propias del campo económico. Acercarnos a la vida social del dinero en un programa local estatal demostró que existe un desplazamiento con respecto a la performatividad pronunciada por los saberes expertos. En ese sentido, al analizar la pluralidad monetaria quedó demostrado que existen desbordes de la teoría performativa experta; es decir, que las premisas del campo económico no clausuran la heterogeneidad de significados que adquiere el dinero en la vida de los sectores populares. Las escenas etnográficas que se incluyen en este artículo revelan, por un lado, los conflictos que deben afrontar los actores locales estatales a la hora de encarnar los significados expertos sobre el dinero y, por otro lado, cómo los adolescentes y los hogares titulares de TM negocian constantemente los significados del dinero con los actores locales estatales.

Producción de condiciones en Villa Asunción demuestra la manera en que los actores locales estatales se debaten entre reproducir los significados expertos acerca de las TM o incorporar sus propias definiciones, e incluso puede que utilicen los significados asociados a esos dineros para clasificar y evaluar a los titulares y a los hogares receptores de TM. Controversias sobre los significados del dinero revela que las personas titulares de TM producen distintas interpretaciones sobre el dinero para evaluar los esquemas de percepción y apreciación que los actores locales estatales poseen sobre el dinero programado de las políticas sociales.

La intención de este artículo, en definitiva, es contribuir a la construcción de una sociología del dinero multisituada (Hornes, 2020). La sociología del dinero que aquí se ha implementado permite mostrar, de forma simultánea, los intersticios y las formas de ensamblaje que existen entre significados del dinero que atraviesan un mundo de prácticas y relaciones sociales plurales. La producción social de las TM es el resultado de un ensamblaje de significados 
plurales del dinero que se suceden en distintos tiempos y espacios sociales e involucran a diferentes tramas de actores que movilizan diversos saberes y producen significados.

El andamiaje metodológico y conceptual que desplegamos en torno a la producción social del dinero de las TM nos permite proponer tres posibles aportes al campo de las políticas sociales.

En primer lugar, el dinero puede servir como indicador cualitativo de las políticas sociales para interpretar qué nuevas formas de inclusión social y desarrollo, qué formas de vulnerabilidad social, exclusión y marginalidad, y qué nuevas relaciones de poder y/o desigualdad, se expresan a través de los significados sociales del dinero. En segundo lugar, podría contribuir a los procesos de formulación y diseño de las TM, en tanto aporta elementos para reinterpretar las definiciones económicas que programan al dinero, reflexionar en torno a la definición de los sujetos titulares de derecho, explorar nuevos requisitos o condicionalidades para las TM, construir nuevos indicadores cualitativos de evaluación, etcétera.

En tercer lugar, las reflexiones sobre el dinero de las TM también pueden aportar a la problematización acerca de los efectos de la implementación de estas nuevas políticas sociales. El dinero puede servir como puerta de entrada para analizar las relaciones sociales entre actores locales estatales y titulares (coerción, control, empatía, etc.), sobre cómo las disputas en torno a sus significados sociales pueden tener incidencia en el acceso a ciertos recursos o a las formas de provisión del bienestar (relaciones de poder y dominación) e, incluso, acerca de cómo puede reproducir jerarquías comunitarias, construir ciertas comunidades de pertenencia o producir nuevas formas de inclusión/ exclusión en el ámbito comunitario.

Esperamos que estas páginas constituyan un aporte a dichas propuestas. 


\section{Q Referencias bibliográficas}

» Barba Solano, Carlos (2011). Hipótesis no comprobadas y espejismo en las políticas de transferencias monetarias condicionadas. En Barba Solano, C., Perspectivas críticas sobre la cohesión social: desigualdad y tentativas fallidas de integración en América Latina. Buenos Aires: Clacso.

» Becker, G. (2008). El capital humano. Madrid: Alianza.

» Blanc, J. (2009). Usages de I argent et pratiques monétaires". En P. Steiner y F. Vatin, (edts.). Traité de sociologie économique (pp. 649-688). París: Presses Universitaries de France.

» Bloch, M. y Parry, J. (eds.) (1989). Money and morality of exchange. Cambridge: Cambridge University Press.

" Cecchini, S. y Martinez, R. (2013) Protección social inclusiva: una mirada integral, un enfoque de derechos. Diálogos Interamericanos de Protección Social. Santiago de Chile: CEPAL.

»Cuillerai, M. (2004) Le capitalisme vertueux ; mondialisation et confiance. Ed. Payot: Paris.

»Cotta, Teresa C., y Paiva, Luis (2010). O Programa Bolsa Família e proteçao social no Brasil. En Bolsa Família 2003-2010: avanços e desafios, volume 1. Brasil: MDS Brasil e IPEA.

»Dapuez, A. (2013) Promissory Prestations: a Yucatec village between ritual exchange and development cash transfers. Tesis de doctorado en Filosofía. Baltymore - Johns Hopkins University.

» Dodd, Nigel. (2014) The Social Life Of Money. Nueva Jersey: Princeton University Press.

» Fontaine, L. (2008). L'économie morale. Pauvreté, crédit et confiance dans l'Europe préindustrielle. Paris: Gallimard.

» Guyer, J. (ed.) (1994). Money Matters: Instability, Values and Social Payments in the Modern History of West African Communities. Londres: Elsevier.

" Guyer, J. (2004). Marginal Gains: Monetary Transactions in Atlantic Africa. Chicago: The University of Chicago Press.

" Hornes, M. (2014). Transferencias condicionadas y sentidos plurales: el dinero estatal en la economía de los hogares argentinos. Antípoda, 18 [Antropología y economía II], 61-83.

"Hornes, M. (2015a). Entre condiciones expertas y negociaciones prácticas: la generización del dinero proveniente de las transferencias monetarias condicionadas. Horizontes antropológicos, 22(45), 77-104.

» Hornes, M. (2015b). Controversias en torno a la construcción pública del dinero. Cuadernos de Antropología Social, 42, 55-71.

"Hornes, Martín (2017). Paradojas de la inclusión: categorías técnicas y evaluaciones morales en un programa de transferencias monetarias condicionadas. Prácticas de Oficio ides, 2(18), 1-14.

" Hornes, M. (2018). Políticas sociales y significados plurales del dinero: la producción social de las transferencias monetarias [Tesis doctoral]. Instituto de Altos Estudios SocialesUniversidad Nacional de San Martín, Buenos Aires, Argentina.

" Hornes, M. (2019). Expertos diseñando el dinero: el caso de los programas de transferencias monetarias. Estudios Sociológicos, 38, 141-171. 
" Hornes, M. (2020) Las tramas del dinero estatal. Sabéres, prácticas y significados del dinero en las políticas sociales argentinas (2008-2015). CABA. TeseoPress.

"Hornes, M y Maglioni, C. (2020). Accesibilidad y políticas sociales en la Argentina reciente: una interpretación desde los saberes expertos en programas de transferencias monetarias. Cuadernos de Trabajo Social de la Universidad Complutense de Madrid, 33, 77-98.

»Lavinas, L. (2017). The Takeover of Social Policy by Financialization. The Brazilian Paradox. Nueva York. Palgrave Macmillan.

" Lo Vuolo, R. (ed.) (2013). The Argentine Universal Child Allowance: Not the Poor but the Unemployed and Informal Workers. En Citizen's Income and Welfare Regimes in Latin America. From Cash Transfers to Rights. (51-66). Nueva York: Palgrave Macmillan.

"Molyneux, M. (2009). Conditional cash transfers: pathways to women's empowerment? Pathways Brief, 5. Recuperado de http://www. pathwaysofempowerment.org/Pathways_ Brief_5.pdf

»Morresi, S. y Vommaro, G. (2011). Saber lo que se hace. Expertos y política en Argentina. Buenos Aires: Prometeo.

»Orléan, A. (2007). La monnaie, opérateur de totalisation. Journal des Anthropologues, 9091, 331-352.

》 Roig, A. (2016). La moneda imposible. Buenos Aires: Fondo de Cultura Económica.

» Thelen, T., Vetters, L. y von Benda-Beckmann, K. (2014). Introduction to Stategraphy. Toward a Relational Antropology of the State. Social Analysis, 58(3), 1-19.

》Théret, B. (2007). La monnaie dévoilee par ses crises. Volume II. Crises monetaires en Russie et en Allemagne au XX siécle. París: Editions de L'ecole des Hautes Etudes en Sciences Sociales.

»Weber, F. y Dufy, C. (2009). Más allá de la Gran División. Sociología, economía y etnografía. Buenos Aires: Antropofagia.

"Wilkis, A y Hornes, M. (2017). Negociando la inclusión al mercado de consumo: Los programas de transferencias condicionadas de dinero y el orden familiar. Civitas, 17(1), 61-78.

»Wilkis, A. (2016). Sociología moral del dinero en el mundo popular. Estudios Sociológicos, XXXIII(99), 553-578.

"Wilkis, A (2017). The Moral Power of Money. Moral and Economy in the poor people life. California: Standord University Press.

"Wilkis, A. (2014). Sociología del crédito y economía de las clases populares. Revista Mexicana de Sociología, 76(2), 225-252.

"Wilkis, A. (2013). Las sospechas del dinero. Moral y economía en el mundo popular. Buenos Aires: Paidós.

"Zelizer, V. (2017). A dollar is not a dollar. Los Angeles Review of books. Recuperado de https://lareviewofbooks.org/article/a-dollar-is-a-dollar-is-not-a-dollar-unmasking-thesocial-and-moral-meanings-of-money/\#!

»Zelizer, V. (2009). La negociación de la intimidad. Buenos Aires: Fondo de Cultura Económica.

» Zelizer, V. (2011). El significado social del dinero. Buenos Aires: Fondo de Cultura Económica. 


\section{Documentos consultados}

» Banco Mundial (2010) World report: Attacking Poverty: Opportunity, Empowerment, and Security. Washington DC. BM.

» Banco Mundial (2009) Transferencias Monetarias Condicionadas: Reduciendo la pobreza actual futura. Washington, DC: Banco Mundial.

» Banco Mundial (2008) Cruces, Guillermo; Moreno, Juan Martin; Ringold, Dena; Rofman, Rafael. (eds.). Los programas sociales en Argentina hacia el bicentenario. Buenos Aires: Banco Mundial.

» CEPAL (2010a), Panorama social de América Latina, 2009 (LC/G.2423-P), Santiago de Chile. Publicación de las Naciones Unidas.

» CEPAL (2010b), La hora de la igualdad: brechas por cerrar, caminos por abrir. Santiago de Chile. Publicación de las Naciones Unidas. 